\section{$T$ cell homeostasis and systemic autoimmunity}

\author{
Argyrios N. Theofilopoulos, \\ Wolfgang Dummer, and Dwight H. Kono
}

The Scripps Research Institute, Department of Immunology, La Jolla, California, USA

Address correspondence to: Argyrios N. Theofilopoulos, Department of Immunology/IMM3, The Scripps Research Institute, 10550 N. Torrey Pines Road, La Jolla, California 92037, USA

Phone: (858) 784-8135; Fax: (858) 784-8361;

E-mail: argyrio@scripps.edu.

J. Clin. Invest. 108:335-340. DOI:10.1172/JCI200112173.

It has recently been documented that survival and homeostasis of peripheral mature $\mathrm{T}$ cells depend on self-recognition. Thus, contrary to previous assumptions, naive $T$ cells apparently require a constant subthreshold signal provided by their engagement with self-MHC/peptide ligands to persist in a quiescent state. In a lymphopenic state, this self-MHC/peptide recognition provides a proliferation-inducing signal and leads to $\mathrm{T}$ cell expansion until the $\mathrm{T}$ cell pool is reestablished to a nearly normal size. Here, we postulate that homeostatic anti-self T cell proliferation may, depending on additional background genes, contribute to systemic autoimmune disease pathogenesis.

Systemic autoimmunity, of which lupus is the prototypic disease, has been extensively investigated, particularly in spontaneous mouse lupus models. Despite key advances in several areas (reviewed in ref. 1), our understanding of the mechanisms leading to a breakdown of tolerance to a wide spectrum of self-molecules remains unclear. Disappointingly, early efforts to demonstrate central $\mathrm{T}$ cell tolerance defects in lupus mice, either with regard to deletions of endogenous superantigen-recognizing $\mathrm{T}$ cells or to conventional self-peptide-recognizing transgenic antigen receptor-expressing $\mathrm{CD}^{+}$or $\mathrm{CD}^{+}$cells, were unsuccessful - even in lupus mice with defects in the proapoptotic Fas pathway, by which activated $T$ cells are eventually deleted. Although peripheral T cells of Fas-defective mice show reduced activation-induced cell death, the reasons for the excessive activation of $\mathrm{T}$ cells therein has not been identified. We argue below that in this and other seemingly unrelated cases, autoimmune manifestations can result from disturbances in $\mathrm{T}$ cell homeostasis that lead to continuous or intermittent $\mathrm{T}$ cell stimulation by self-MHC/peptide ligands.

\section{Homeostatic $\mathrm{T}$ cell anti-self proliferation}

During intrathymic development, a small fraction of $\mathrm{T}$ cells expressing receptors of sufficient affinity for
self-MHC/peptide ligands is positively selected, survives, and differentiates, whereas the majority of $\mathrm{T}$ cells with little or no affinity for such ligands undergoes apoptotic death by neglect. Thereafter, those positively selected cells with dangerously high affinity for selfMHC/peptide ligands are also deleted by apoptosis (negative selection), and only a small fraction (3-5\%) is released to the periphery to constitute the mature $\mathrm{T}$ cell pool (reviewed in ref. 2).

According to previously accepted views, naive $\mathrm{T}$ cells that survive these somatic selection processes persist in a dormant state within the confines of the secondary lymphoid organs, unaware of self-antigens until engaged by activated antigen-presenting cells displaying foreign antigenic peptides. This assumption has recently been challenged by several groups, who have shown that low-grade recognition of self-MHC/peptide ligands and delivery of covert signals - those that do not induce proliferation, as do conventional antigen-provoked, overt signals - are requirements for long-term survival of naive $\mathrm{T}$ cells in the periphery (Figure 1). Thus, in MHC class II-deficient mice manipulated to express the class II molecules only in the thymus, exported $\mathrm{CD}^{+}$cells do not survive long in the class II-barren periphery $(3,4)$, although this population can be rescued upon transfer of class $\mathrm{II}^{+}$dendritic cells (5). Similarly, naive $\mathrm{H}-\mathrm{Y}$-specific $\mathrm{T}$ cell receptor transgenic (TCR transgenic) $\mathrm{CD}^{+}$cells do not survive after adoptive transfer into an MHC class I-deficient host or in a host carrying an MHC class I molecule different from the one on which these cells had been selected $(6,7)$. The molecular events associated with survival-promoting interactions have not been fully defined, but work with naive $\mathrm{T}$ cells derived from $\mathrm{MHC}$ class $\mathrm{II}^{+/+}$environments shows partial phosphorylation of the $\mathrm{T}$ cell receptor complex protein $\mathrm{CD} 3-\varsigma(\mathrm{pp} 21)$ as well as recruitment of the ZAP-70 kinase to this complex (8). In contrast to proliferation-inducing signals, however, there is no recruitment of the p56 lck kinase (9). It should be noted that, despite considerable supporting evidence, some recent studies have called into question the strict necessity of self-MHC/peptide ligand recognition for the survival of naive $\mathrm{CD}^{+}$cells in the periphery $(10,11)$.

Further studies have also established that self-recognition is important to the homeostatic process that maintains the near-constant overall size of the peripheral $\mathrm{T}$ cell pool, which is established at a young age. Homeostatic adjustment of $T$ cell numbers is necessary at the final stage of an immune response, during which time the vast majority of the specific effector cells die by apoptosis. Such an adjustment will also occur after the onset of lymphopenia induced by a variety of insults (e.g., viral infections, toxic agents, irradiation, cytotoxic drugs). Restoration of the size of the original $\mathrm{T}$ cell pool following severe $\mathrm{T}$ cell defi- 
a

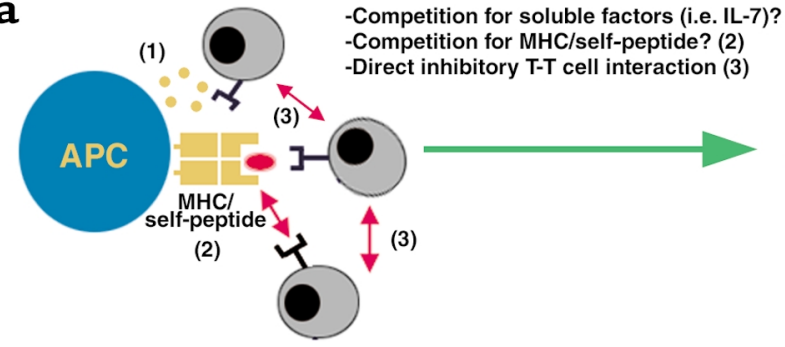

$-0$

\begin{abstract}
-No upregulation of activation markers
-No homeostatic expansion
\end{abstract} -Low basal TCR engagement

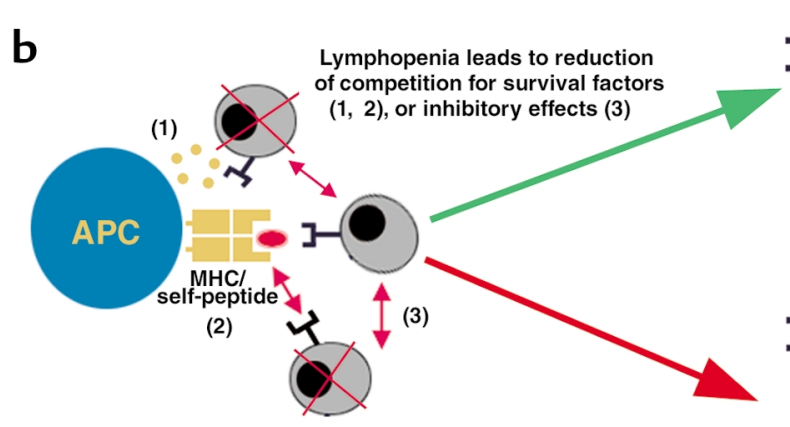

-Appropriate genetic background -Duration, intensity, frequency of lymphopenia

-Inflammation

\section{0}

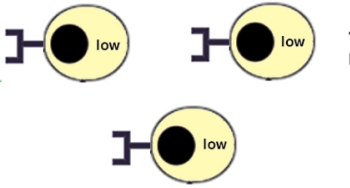

nnocuous homeostatic T cell expansion

-Upregulation of some activation markers (i.e. CD44, CD122)

\section{Figure 1}

Regulation of naive $T$ cell homeostasis in the periphery and the potential relevance for the development of autoimmunity. Positive and negative selection in the thymus creates a repertoire of T cells with a broad spectrum of affinities to self, from low self-affinity (just strong enough to escape from death by neglect) to comparatively high self-affinity, which is, however, not strong enough to undergo negative selection. In a T cell-sufficient individual, the bulk of naive T cells needs continuous signals by self-MHC/self-peptide complexes to survive in a quiescent state. How the presence of surrounding T cells prevents naive T cells from uncontrolled proliferation is currently unknown. Competition for soluble stimulatory factors (labeled " 1 " in figure), for contact with self-MHC/self-peptide complexes (labeled " 2 "), or a direct inhibitory effect between T cells themselves (labeled " 3 ") are possible mechanisms. Even while resting, a certain degree of T cell receptor engagement is measurable. Reduction of T cell numbers leads to spontaneous homeostatic T cell expansion, either by increased availability of stimulatory factors (labeled " 1 ," " 2 ") or by reduction of inhibitory impulses (labeled "3"). Homeostasis-driven proliferation leads to upregulation of some activated/memory markers. This proliferation usually occurs innocuously, without harm to normal individuals under physiologic circumstances. Either through constitutive T cell depletion or repeated episodes of $T$ cell depletion, a T cell repertoire might be skewed to more T cells with high self-affinity. In this scenario, when homeostatic T cell expansion occurs, such cells, in conjunction with the appropriate genetic background and/or coexisting inflammation, might be overstimulated. In some models, such $\mathrm{T}$ cells have been shown to hyper-respond and acquire effector function. This provides the basis for the development of autoimmunity.

ciency has long been known to be accomplished by spontaneous expansion of the spared T cells, even in the absence of any contribution from the thymus (12). Accordingly, homeostatic proliferation of $\mathrm{T}$ cells has been observed upon adoptive transfer of small numbers of cells into syngeneic lymphopenic $n u / n u$, SCID, RAG-deficient, TCR $\alpha$-chain-deficient, or sublethally irradiated hosts (13-15). Homeostatic proliferation of $\mathrm{T}$ cells is polyclonal and occurs in the absence of deliberate immunization.

Recent studies have now made it clear that homeostatic proliferation of $T$ cells is mediated by, and dependent upon, recognition of self-MHC/peptide ligands. Accordingly, naive $\mathrm{CD} 4^{+}$cells from wild-type $\mathrm{B} 6$ mice do not undergo efficient homeostatic proliferation when transferred to H-2M-deficient, syngeneic T cell-depleted mice, which express MHC class II molecules loaded almost exclusively with a single species of peptide (16, 17). In contrast, naive $\mathrm{CD} 4^{+}$cells from $\mathrm{H}-2 \mathrm{M}$-deficient mice that were positively selected to this peptide proliferated well in T cell-depleted H-2M-deficient hosts, again arguing that lymphopenia-induced homeostatic
$\mathrm{T}$ cell proliferation in the periphery depends on recognition of self-peptides identical to those used in their positive selection. Additional support for this model comes from experiments involving T cell-depleted mice engineered to express MHC class I molecules that were loaded with specific peptides: TCR transgenic $\mathrm{CD}^{+}$ cells transfused into these animals proliferated only when the host expressed a peptide that induced the positive selection of the $\mathrm{T}$ cells, but not when the host expressed an irrelevant peptide (18).

Together, these findings indicate that the weak interaction mediated by self-MHC/peptide recognition

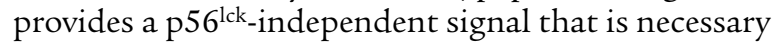
for survival in a resting state, but that under lymphopenic conditions, this signal instead becomes p56 lck-dependent, is translated differently, and leads to strong proliferation (reviewed in 19-21; Figure 1). In another recent study (22), it was shown that $\mathrm{T}$ cells have to enter intact $T$ cell compartments in secondary lymphoid tissues in order for homeostatic proliferation to occur. Experiments to clarify why homeostatic proliferation does not occur in a nonlymphopenic 
state found that a large number of "bystander" $T$ cells coinjected with a small number of syngeneic $\mathrm{T}$ cells (differentiated by allelic markers and intracellular dyes) can inhibit expansion of the latter in a lymphopenic host. Of interest, naive $T$ cells can act as bystander inhibitors, whereas activated/memory phenotype $T$ cells are inefficient inhibitors. Crucially, bystander naive $T$ cells in these experiments did not require signaling through the TCR, nor did they need to undergo homeostatic proliferation themselves to inhibit homeostatic proliferation of the small numbers of $T$ cells that were coinfused. These findings suggest that $\mathrm{T}$ cell homeostasis is likely regulated either by non-MHC-stimulating factors, such as stromal cell-derived cytokines (i.e., IL-7, IL-12) $(23,24)$, or by direct intercellular contact. Several studies $(6,16,17$, 24-26) have also shown that although all typical $\mathrm{T}$ cells are thought to require positive selection by self$\mathrm{MHC} /$ peptide ligands in the thymus, only a fraction $(\sim 30 \%)$ can undergo homeostatic proliferation in the periphery. The reasons for this selective response remain unclear, but it may be that some positiveselecting peptides are absent from the periphery or that expansion is restricted to cells with higher selfaffinity. Recent experiments with transgenic $\mathrm{T}$ cells favor the second possibility (27).

The relevance of homeostatic anti-self proliferation of naive $T$ cells to autoimmunity rests to a great extent on whether the proliferating cells acquire effector function and differentiate to the activated/memory phenotype. Several studies in which TCR transgenic $\mathrm{CD}^{+}$or $\mathrm{CD}^{+}$cells were transfused to lymphopenic syngeneic hosts appear to provide an affirmative answer $(11,25-29)$. Proliferating TCR transgenic $\mathrm{CD}^{+}$ cells kill target cells ex vivo in a peptide- and TCRdependent manner, and express IFN- $\gamma$ after stimulation with anti-CD3. In vivo, they also acquire several, but not all, phenotypic markers of activated/memory $\mathrm{T}$ cells (upregulation of CD44, CD122, and CD132, and downregulation of CD45RB, but no upregulation of conventional antigen-induced early activation markers CD69, CD71, and CD25 or downregulation of CD62L). Whereas acquisition of CD44 is slower in homeostatically proliferating $\mathrm{T}$ cells than in cognate antigen-driven proliferation, expression of CD122 and CD132 depends strictly on the number of divisions, regardless of whether they are homeostasis- or antigen-driven (29). In two of these studies $(11,28)$, even polyclonal $\mathrm{CD}^{+}$or $\mathrm{CD}^{+}$cells undergoing homeostasis-driven proliferation also acquired activated $/ \mathrm{mem}$ ory phenotypes, and such polyclonal $\mathrm{CD}^{+}$cells were able to rapidly secrete IFN- $\gamma$ after anti-CD3 stimulation, and to kill ConA-coated syngeneic targets; control naive $\mathrm{CD}^{+}$cells were devoid of such activities.

Following reestablishment of nearly normal numbers of $\mathrm{T}$ cells and cessation of proliferation, the majority of the expanded cells retain the activated/memory phenotype, but a small percentage revert to the naive phenotype $(11,25,26,29)$. Maintenance of or reversion to the activated/memory phenotype may depend on the initial affinity for selfMHC/peptide complexes. Thus, cells with high affinity may retain the activated/memory phenotype, while those with low affinity lose it (20). Therefore, it has been concluded that "memory" phenotype $T$ cells in an individual may not all be true foreign antigenexperienced cells, but could include naive cells "masquerading" as memory cells (29).

\section{Homeostatic $T$ cell proliferation in models of induced systemic autoimmunity}

Several findings support the role of homeostatic antiself $\mathrm{T}$ cell proliferation in the pathogenesis of autoimmunity, including autoimmune disease manifestations in mice and rats following neonatal thymectomy, discontinuation of cyclosporin treatment, irradiation, retroviral infections, or exposure to certain xenobiotics. Other examples may be spontaneous autoimmunity in genetically lymphopenic BB rats, and autoimmunity upon transfer of lymphocytes into RAG-deficient, $n u / n u$, or SCID mice (Table 1).

Neonatally thymectomized mice of several, but not all, backgrounds develop a strain-dependent spectrum of autoimmune reactions against multiple organs, as judged by the presence of $\mathrm{T}$ cell infiltrates, inflammation, and appearance of autoantibodies (reviewed in refs. 30,31). There is a strict temporal relationship between the day of thymectomy (third to seventh day of life) and the development of autoimmunity. Importantly, inoculation of $\mathrm{T}$ cells from adult euthymic animals can prevent disease in thymectomized syngeneic mice. This inhibition appears to be mediated primarily by a thymus-derived, naturally unresponsive (anergic) $\mathrm{CD}^{+} \mathrm{CD}_{25} 5^{+}$(IL-2R $\alpha$-chain) population. These cells comprise approximately $5-10 \%$ of thymic and peripheral $T$ cells, are functionally distinct from other activated $\mathrm{T}$ cells, and mediate suppression in an antigen-nonspecific manner. Activation of $\mathrm{CD} 4^{+} \mathrm{CD} 25^{+}$ regulatory $\mathrm{T}$ cells does not seem to require CD28 costimulation, but their generation appears to depend on CD40-CD40L interactions. Recent studies suggest that these cells suppress inflammatory and autoimmune manifestations by a constitutive expression of CTLA-4, a molecule that inhibits CD28/B7-mediated costimulation $(32,33)$. It is of interest that CTLA-4-deficient mice develop a severe lymphoproliferative disorder and die from autoimmune-like disease within 1 month of birth (34). Autoimmunity with multiorgan involvement and even lupus-like manifestations have also been reported in adult athymic $n u / n u$ mice following

\section{Table 1}

Conditions in which homeostatic anti-self T cell proliferation may contribute to the appearance of autoimmune manifestations

\section{Neonatal thymectomy}

Discontinuation of cyclosporin treatment

Irradiation

Retroviral infections

Exposure to cytotoxic drugs

Exposure to xenobiotics

Lymphopenic BB rats

Lymphocyte transfer into $n u / n u$, RAG-deficient, or SCID mice 
inoculation with either spleen cell suspension from 3 -day-old euthymic $\mathrm{nu}^{+/-}$mice or $\mathrm{CD} 4^{+} \mathrm{CD} 25^{+}$-depleted spleen suspension from adult $n u^{+/-}$mice $(30,31)$.

Autoimmunity associated with neonatal thymectomy has been attributed to the absence of the regulatory $\mathrm{CD} 4^{+} \mathrm{CD} 25^{+}$subset (which appears to develop later in ontogeny), inefficient deletion of self-reactive $\mathrm{T}$ cells due to low MHC expression in the neonatal thymus, and/or late developmental thymic expression of certain self-molecules. In view of the findings pertaining to self-MHC/peptide-mediated homeostatic expansion of $\mathrm{T}$ cells, we propose that another contributing factor in autoimmunity associated with neonatal thymectomy and related models may be a dearth of peripheral $\mathrm{T}$ cells, leading to a strong anti-self homeostatic proliferation that fills this "empty space." We also suggest that inhibition of neonatal thymectomy-induced autoimmunity by supplementation with large numbers of adult $\mathrm{T}$ cells may depend on the restoration of the $\mathrm{T}$ cell pool and the consequent curtailment of homeostatic proliferation. The regulatory $\mathrm{CD} 4^{+} \mathrm{CD} 25^{+} \mathrm{T}$ cells may also exert their autoimmunity-inhibiting effect by suppressing homeostatic antiself $T$ cell proliferation.

Another potential example of autoimmunity caused by disturbance of lymphocyte homeostasis and subsequent attempts to reestablish the immune system is observed in certain rat and mouse strains treated neonatally with cyclosporin (CsA), or as adults with lethal irradiation and bone marrow reconstitution followed by treatment with, and subsequent withdrawal of, CsA (reviewed in ref. 35). Development of this disease requires the presence of a thymus and the elimination of peripheral $\mathrm{T}$ cells by irradiation. The disease is mediated by self-MHC class II/peptide-reactive IL-2 and IFN- $\gamma$-producing T cells, and can be transferred to syngeneic irradiated hosts. Once again, transfer of the disease can be prevented by coinfusion of large numbers of spleen $T$ cells from normal adult mice. The precise mechanisms by which this immunosuppressive drug can paradoxically induce autoimmunity remain largely unexplained. Although some studies have reported interference with apoptosis and intrathymic negative selection, others have failed to confirm such a defect, but report inhibition of suppressor $T$ cell action or prevention of peripheral T cell clonal anergy $(35,36)$. We suggest that a more appropriate explanation may be homeostatic anti-self $\mathrm{T}$ cell proliferation triggered by the severe lymphopenia that follows irradiation and cyclosporin treatment. Indeed, cyclosporin has been shown to cause thymic involution and a severe block in the maturation of double-positive $\left(\mathrm{CD}^{+} \mathrm{CD}^{+}\right)$thymocytes and a consequent drop in the output of recent $T$ cell emigrants to the periphery (37).

Several other instances of autoimmune disease development, presumably caused by perturbations in lymphocyte homeostasis, have been reported. For example, high-dose, fractionated, total lymphoid irradiation of mice can induce multiorgan autoimmunity with characteristics dependent on radiation dose, extent of lymphoid irradiation, and the genetic background of the mouse (38). Radiation-induced tissue damage is not the primary cause of these disorders, because irradiation of the target organs alone fails to elicit autoimmunity, and shielding them from irradiation does not prevent it. This syndrome is also prevented by inoculation of splenic $\mathrm{CD}^{+}$cells from syngeneic nonirradiated mice, a treatment that probably inhibits homeostatic proliferation by restoring the $\mathrm{T}$ cell pool. Thus, this disease appears to result from lymphopenia and subsequent elicitation of anti-self homeostatic responses. Similar phenomena have been reported with certain mouse strains infected neonatally with a $\mathrm{CD}^{+}$-depleting mouse $\mathrm{T}$ lymphotropic virus (39). As with the other models discussed above, inoculation of peripheral $\mathrm{CD} 4^{+}$cells from syngeneic, noninfected adult mice prevented the development of autoimmunity. A similar mechanism may be operative in the unexplained autoimmune manifestations characteristic of many HIV-infected individuals with AIDS (reviewed in ref. 40). Certain xenobiotics, such as mercuric chloride $\left(\mathrm{HgCl}_{2}\right)$ and gold, also induce autoimmunity resembling lupus in some rat and mouse strains (reviewed in ref. 41). The mechanism of xenobiotic-induced autoimmunity is unknown, but this condition is characterized by excessive in vivo $\mathrm{T}$ cell proliferation and anti-self-MHC class II reactivity; therefore it is likely that disease in this model may also be attributed to disturbances in $\mathrm{T}$ cell homeostasis.

A few final examples in which low levels of $\mathrm{T}$ cells appear to result in autoimmune diseases: First, as a result of an as-yet undefined genetic defect, $\mathrm{BB} / \mathrm{W}$ rats exhibit excessive $\mathrm{T}$ cell apoptosis and lymphopenia associated with autoimmune manifestations and diabetes (42). Likewise, SCID mice transplanted with coisogenic $\mathrm{CD} 4^{+} \mathrm{CD} 45 \mathrm{RB}{ }^{\text {hi }}$ naive lymph node $\mathrm{T}$ cells develop autoimmune colitis, which can be prevented by coinjection of high IL-10- and low IL-2-producing $\mathrm{CD}^{+} \mathrm{T}$ cells (43). Finally, a similar inflammatory bowel disease has been reported in RAG-deficient mice injected with $\mathrm{CD} 4^{+} \mathrm{CD} 45 \mathrm{RB}{ }^{\text {hi }}$ cells (44). The transferred cells acquired an activated phenotype and show high proliferative capacity.

\section{Homeostatic $\mathrm{T}$ cell proliferation in spontaneous systemic autoimmunity}

Several findings in predisposed mice appear to support the concept that primary or secondary homeostatic lymphocyte perturbations contribute to the pathogenesis of spontaneous lupus. These mice show premature thymic involution, which can lead to reduced export of naive $T$ cells, excessive $T$ cell activation leading to replicative senescence, reduced number and/or function of regulatory $\mathrm{T}$ cells, and in some strains, lymphopenia induced by anti-lymphocyte autoantibodies or increased apoptosis (Table 2).

Thymic atrophy in lupus-prone mouse strains is first evident as a loss of cortical thymocytes, which may later be accompanied by medullary degeneration (reviewed in ref. 1). The thymic atrophy associated with abnormal fine structure appears by the age of 3-4 months in female $(\mathrm{NZB} \times \mathrm{NZW}) \mathrm{F}_{1}$ mice, which, by 6-7 months of age, have lost $70-90 \%$ of their cortices. In BXSB males and MRL-Faspr mice of both sexes, thymic atrophy and cystic necrosis appear by the age of 2 months and 


\section{Table 2}

Postulated mechanisms by which homeostatic anti-self $\mathrm{T}$ cell proliferation may contribute to lupus pathogenesis

Premature thymic involution

Accumulation of activated/memory T cells in a replicative senescence state Reduced numbers or function of regulatory $T$ cells

Lymphopenia due to anti-lymphocyte autoantibodies

Lymphopenia due to increased apoptosis

progress to a complete loss of the cortical areas by 4.5 and 3.5 months, respectively. Ultrastructural studies and immunocytochemistry with mAb's to thymic constituents have shown considerable abnormalities, including dramatic alterations in microarchitecture, decreases in subcapsular and medullary epithelia, free spaces ("cortical holes"), decreased thymocyte frequencies, and altered composition (45). Although these changes have been considered manifestations of premature aging, they differ considerably from those seen in normal aging mice. The relevance of these findings to our hypothesis lies in the possibility that intrinsic thymic defects in $T$ cell maturation may lead to a lymphocyte deficit in the periphery, and consequently, to the initiation of a homeostatic, yet deleterious, anti-self response.

Another possible means of homeostatic disturbance in lupus is through continuous $T$ cell stimulation by ever-present self-antigens, and attainment of a state of replicative senescence wherein such cells may be unable to act as efficient inhibitors of homeostatic proliferation. Indeed, mice (and humans) with lupus show an expedited accumulation of activated/memory phenotype cells with clonal expansions (46). Moreover, most of the accumulated activated/memory phenotype $\mathrm{CD} 4^{+} \mathrm{CD} 44^{\text {hi }}$ cells of male BXSB mice and double-negative (DN CD4-CD8-) CD44hi cells of MRL-Fas ${ }^{\text {lpr }}$ mice are arrested at the $\mathrm{G} 1$ phase of the cell cycle, express high levels of cyclin kinase inhibitors, and are refractory to apoptosis (46), all characteristics of cells in replicative senescence (47). Such cells may be inefficient inhibitors of naive $\mathrm{T}$ cell homeostatic proliferation, since such proliferation can be inhibited by large numbers of naive, but not activated, T cells (22).

Lymphopenia, which occurs in a high percentage of lupus patients in the acute phases, may also trigger homeostatic anti-self $\mathrm{T}$ cell proliferation. The absolute levels of lymphocytes are reduced in this disorder, as are the percentages of $\mathrm{T}$ and $\mathrm{B}$ cells. Patients not undergoing prednisolone treatment also show a marked reduction of $\mathrm{CD}^{+}$cells. Lymphopenia in lupus has primarily been attributed to anti-lymphocyte autoantibodies and/or increased activationinduced apoptosis via the Fas/FasL system. Anti-lymphocyte autoantibodies are also present in several of the lupus-predisposed mouse strains.

Apart from lupus, homeostatic anti-self proliferation of $\mathrm{T}$ cells may play a role in other autoimmune conditions wherein the composition of the peripheral repertoire and other factors may dictate a different clinical outcome. Recent studies have shown that patients with rheumatoid arthritis exhibit a premature decline in thymic output and a compensatory expansion of peripheral T cells (48). It has therefore been hypothesized that these phenomena may lead to a repertoire contraction that may favor T cells with autoreactive potential (49).

\section{Summary}

The exact mechanisms by which homeostatic anti-self proliferation of peripheral $\mathrm{T}$ cells is controlled are not yet well understood. Nevertheless, evidence exists that self-MHC/peptide recognition, necessary for $\mathrm{T}$ cell survival in a resting state, may become overt under certain conditions associated with lymphopenia and lead to $T$ cell expansion and generation of effector cells (Figure 1). Several factors may influence whether this expansion is beneficial or detrimental to the host, including genetic background, intensity, frequency and duration of lymphocyte disturbances, and previous inflammatory damage of the target organs. We further postulate that if the $T$ cell repertoire and expanded clones primarily have low self-affinity, then the expansion will be largely innocuous, whereas if composed of cells with high self-affinity, clinically evident autoaggression may be the outcome. Validation of this hypothesis may provide a new paradigm for understanding autoimmune syndromes, including lupus.

\section{Acknowledgments}

This is publication number 13818-IMM from the Department of Immunology, The Scripps Research Institute (La Jolla, California, USA). The authors thank Charles Surh for manuscript review and M. Kat Occhipinti-Bender for editorial assistance. Space limitations necessitated citing reviews rather than original articles in many instances.

1. Theofilopoulos, A.N., and Kono, D.H. 1998. Murine lupus models: genespecific and genome-wide studies. In Systemic lupus erythematosus. 3rd edition. R.G. Lahita, editor. Academic Press Inc. San Diego, California, USA. $145-181$

2. Kisielow, P., and von Boehmer, H. 1995. Development and selection of T cells: facts and puzzles. Adv. Immunol. 58:87-209.

3. Takeda, S., Rodewald, H.R., Arakawa, H., Bluethmann, H., and Shimizu, T. 1996. MHC class II molecules are not required for survival of newly generated CD4+ T cells, but affect their long-term life span. Immunity. 5:217-228.

4. Kirberg, J., Berns, A., and von Boehmer, H. 1997. Peripheral T cell survival requires continual ligation of the $\mathrm{T}$ cell receptor to major histocompatibility complex-encoded molecules. J. Exp. Med. 186:1269-1275.

5. Brocker, T. 1997. Survival of mature CD4 T lymphocytes is dependent on major histocompatibility complex class II-expressing dendritic cells. J. Exp. Med. 186:1223-1232.

6. Tanchot, C., Lemonnier, F.A., Perarnau, B., Freitas, A.A., and Rocha, B. 1997. Differential requirements for survival and proliferation of CD8 naive or memory T cells. Science. 276:2057-2062.

7. Nesic, D., and Vukmanovic, S. 1998. MHC class I is required for peripheral accumulation of CD8+ thymic emigrants. J. Immunol. 160:3705-3712.

8. Witherden, D., et al. 2000. Tetracycline-controllable selection of CD4(+) T cells: half-life and survival signals in the absence of major histocompatibility complex class II molecules. J. Exp. Med. 191:355-364.

9. Seddon, B., Legname, G., Tomlinson, P., and Zamoyska, R. 2000. Longterm survival, but impaired homeostatic proliferation, of naive $T$ cells in the absence of p56lck. Science. 290:127-131.

10. Dorfman, J.R., Stefanova, I., Yasutomo, K., and Germain, R.N. 2000. CD4+ T cell survival is not directly linked to self-MHC-induced TCR signaling. Nat. Immunol. 1:329-335.

11. Clarke, S.R., and Rudensky, A.Y. 2000. Survival and homeostatic proliferation of naive peripheral CD4+ T cells in the absence of self peptide:MHC complexes. J. Immunol. 165:2458-2464.

12. Mackall, C.L., Hakim, F.T., and Gress, R.E. 1997. Restoration of T-cell homeostasis after T-cell depletion. Semin. Immunol. 9:339-346.

13. Bell, E.B., Sparshott, S.M., Drayson, M.T., and Ford, W.L. 1987. The sta- 
ble and permanent expansion of functional $\mathrm{T}$ lymphocytes in athymic nude rats after a single injection of mature $\mathrm{T}$ cells. J. Immunol. 139:1379-1384.

14. Rocha, B., Dautigny, N., and Pereira, P. 1989. Peripheral T lymphocytes: expansion potential and homeostatic regulation of pool sizes and CD4/CD8 ratios in vivo. Eur. J. Immunol. 19:905-911.

15. Tanchot, C., Rosado, M.M., Agenes, F., Freitas, A.A., and Rocha, B. 1997. Lymphocyte homeostasis. Semin. Immunol. 9:331-337.

16. Ernst, B., Lee, D.S., Chang, J.M., Sprent, J., and Surh, C.D. 1999. The peptide ligands mediating positive selection in the thymus control $\mathrm{T}$ cell survival and homeostatic proliferation in the periphery. Immunity. 11:173-181.

17. Viret, C., Wong, F.S., and Janeway, C.A., Jr. 1999. Designing and maintaining the mature TCR repertoire: the continuum of self-peptide:selfMHC complex recognition. Immunity. 10:559-568.

18. Goldrath, A.W., and Bevan, M.J. 1999. Low-affinity ligands for the TCR drive proliferation of mature CD8+ T cells in lymphopenic hosts. Immunity. 11:183-190.

19. Goldrath, A.W., and Bevan, M.J. 1999. Selecting and maintaining a diverse T-cell repertoire. Nature. 402:255-262.

20. Surh, C.D., and Sprent, J. 2000. Homeostatic T cell proliferation. How far can T cells be activated to self-ligands? J. Exp. Med. 192:F9-F14.

21. Marrack, P., et al. 2000. Homeostasis of alpha beta TCR+ T cells. Nat. Immunol. 1:107-111.

22. Dummer, W., Ernst, B., LeRoy, E., Lee, D.-S., and Surh, C.D. 2001. Autologous regulation of naive $\mathrm{T}$ cell homeostasis within the $\mathrm{T}$ cell compartment. J. Immunol. 166:2460-2468.

23. Schluns, K.S., Kieper, W.C., Jameson, S.C., and Lefrancois, L. 2000. Interleukin-7 mediates the homeostasis of naive and memory CD8 T cells in vivo. Nat. Immunol. 1:426-432.

24. Kieper, W.C., Prlic, M., Schmidt, C.S., Mescher, M.F., and Jameson, S.C 2001. IL-12 enhances CD8 T cell homeostatic expansion. J. Immunol. 166:5515-5521.

25. Oehen, S., and Brduscha-Riem, K. 1999. Naive cytotoxic T lymphocytes spontaneously acquire effector function in lymphocytopenic recipients: a pitfall for T cell memory studies? Eur. J. Immunol. 29:608-614.

26. Goldrath, A.W., Bogatzki, L.Y., and Bevan, M.J. 2000. Naive T cells transiently acquire a memory-like phenotype during homeostasis-driven proliferation. J. Exp. Med. 192:557-564.

27. Ge, Q., Rao, V.P., Cho, B.K., Eisen, H.N., and Chen J. 2001. Dependence of lymphopenia-induced $\mathrm{T}$ cell proliferation on the abundance of peptide/MHC epitopes and strength of their interaction with T cell receptors. Proc. Natl. Acad. Sci. USA. 98:1728-1733.

28. Cho, B.K., Rao, V.P., Ge, Q., Eisen, H.N., and Chen, J. 2000. Homeostasis-stimulated proliferation drives naive $\mathrm{T}$ cells to differentiate directly into memory T cells. J. Exp. Med. 192:549-556.

29. Murali-Krishna, K., and Ahmed, R. 2000. Cutting edge: naive T cells masquerading as memory cells. J. Immunol. 165:1733-1737.

30. Sakaguchi, S. 2000. Regulatory T cells: key controllers of immunologic self-tolerance. Cell. 101:455-458.

31. Shevach, E.M. 2000. Regulatory T cells in autoimmunity. Annu. Rev. Immunol. 18:423-449.
32. Read, S., Malmstrom, V., and Powrie, F. 2000. Cytotoxic T lymphocyteassociated antigen 4 plays an essential role in the function of CD25(+)CD4(+) regulatory cells that control intestinal inflammation. J. Exp. Med. 192:295-302.

33. Takahashi, T., et al. 2000. Immunologic self-tolerance maintained by CD25(+)CD4(+) regulatory $\mathrm{T}$ cells constitutively expressing cytotoxic $\mathrm{T}$ lymphocyte-associated antigen 4. J. Exp. Med. 192:303-310.

34. Tivol, E.A., et al. 1995. Loss of CTLA-4 leads to massive lymphoproliferation and fatal multiorgan tissue destruction, revealing a critical negative regulatory role of CTLA-4. Immunity. 3:541-547.

35. Bucy, R.P., Xu, X.Y., Li, J., and Huang, G. 1993. Cyclosporin A-induced autoimmune disease in mice. J. Immunol. 151:1039-1050.

36. Prud'homme, G.J., and Vanier, L.E. 1993. Cyclosporine, tolerance, and autoimmunity. Clin. Immunol. Immunopathol. 66:185-192.

37. Beijleveld, L.J., Damoiseaux, J.G., and Van Breda Vriesman, P.J. 1995. Differential effects of $\mathrm{X}$-irradiation and cyclosporin-A administration on the thymus with respect to the generation of cyclosporin-A-induced autoimmunity. Dev. Immunol. 4:127-138.

38. Sakaguchi, N., Miyai, K., and Sakaguchi, S. 1994. Ionizing radiation and autoimmunity. Induction of autoimmune disease in mice by high dose fractionated total lymphoid irradiation and its prevention by inoculating normal T cells. J. Immunol. 152:2586-2595.

39. Morse, S.S., Sakaguchi, N., and Sakaguchi, S. 1999. Virus and autoimmunity: induction of autoimmune disease in mice by mouse $\mathrm{T}$ lymphotropic virus (MTLV) destroying CD4+ T cells. J. Immunol. 162:5309-5316

40. Ende, N., and Schwartz, R.A. 1997. Autoimmunity and AIDS. A commentary. J. Exp. Med. 28:273-274.

41. Pollard, K.M., and Hultman, P. 1997. Effects of mercury on the immune system. Met. Ions Biol. Syst. 34:421-440.

42. Zadeh, H.H., Greiner, D.L., Wu, D.Y., Tausche, F., and Goldschneider, I. 1996. Abnormalities in the export and fate of recent thymic emigrants in diabetes-prone BB/W rats. Autoimmunity. 24:35-46.

43. Groux, H., et al. 1997. A CD4+ T cell subset inhibits antigen-specific Tcell responses and prevents colitis. Nature. 389:737-742.

44. Annacker, O., Burlen-Defranoux, O., Pimenta-Araujo, R., Cumano, A., and Bandeira, A. 2000. Regulatory CD4 T cells control the size of the peripheral activated/memory CD4 T cell compartment. J. Immunol. 164:3573-3580.

45. Takeoka, Y., et al. 1995. Thymic microenvironmental abnormalities in MRL/MP-lpr/lpr, BXSB/MpJ Yaa and C3H HeJ-gld/gld mice. J. Autoimmun. 8:145-161.

46. Sabzevari, H., Propp, S., Kono, D.H., and Theofilopoulos, A.N. 1997. G1 arrest and high expression of cyclin kinase and apoptosis inhibitors in accumulated activated/memory phenotype CD4+ cells of older lupus mice. Eur. J. Immunol. 27:1901-1910.

47. Campisi, J. 1996. Replicative senescence: an old lives' tale. Cell. 84:497-500.

48. Koetz, K., et al. 2000. T cell homeostasis in patients with rheumatoid arthritis. Proc. Natl. Acad. Sci. USA. 97:9203-9208.

49. Goronzy, J.J., and Weyand, C.M. 2001. Thymic function and peripheral T-cell homeostasis in rheumatoid arthritis. Trends Immunol. 22:251-255. 